\title{
CRITICAL FEATURES IN HUMAN MOTION SIMULATION FOR ERGONOMIC ANALYSIS
}

\author{
Matthew P. Reed, Don B. Chaffin, and Julian Faraway \\ University of Michigan
}

\begin{abstract}
Digital human figure models (DHM) are increasingly the tools of choice for assessments of the physical ergonomics of products and workplaces. Software representations of users and workers are used to visualize people performing tasks of interest. Analyses have usually focused on clearance and reach in static postures, not because the actual tasks are static, but rather because DHM have lacked robust, accurate motion simulation capability. Research is underway at many institutions to develop improved motion simulation methods, drawing on a wide variety of methodologies from fields such as computer graphics, kinesiology, motor control, and robotics. Experience in the Human Motion Simulation Laboratory at the University of Michigan suggests that conventional metrics of accuracy for posture and movement prediction do not adequately capture the aspects of human movement that are most important for ergonomic analysis. This paper identifies and justifies a set of these critical features.
\end{abstract}

\section{INTRODUCTION}

Most commercial figure models userd for ergonomic analysis, including widely used software packages such as Jack, Ramsis, and Safework, are capable of simulating taskoriented human motion. This capability might be more accurately termed animation rather than simulation, because the motions are usually produced as interpolations between postures that are manually generated by the software user. Many motion simulation approaches have been published, but mainstream digital human modeling software packages that are used for ergonomics analysis still do not have general, robust motion simulation capability. Barriers to implementation of research results into commercial models include incompatibilities in kinematic linkages, a lack of robustness in the simulation algorithms (applicable to only a small class of motions, for example), and prohibitive computation requirements. In addition to these issues, one of the major challenges in creating motion simulation algorithms is defining and measuring the accuracy of motion simulation.

Most papers presenting a motion simulation algorithm have focused on Euler or Cardan angles defined at joints of a kinematic linkage (e.g., Zhang et al. 1998). The algorithms predict angle trajectories, which are applied to a kinematic linkage to simulate motion of some or all of the body. The accuracy of the motion is quantified by the deviations of measured and predicted joint angles, comparing the simulations to the measured motions of study participants. A typical metric is the root-mean-square error of a joint angle trajectory, perhaps summed across joints.

Experience with implementing motion prediction algorithms in commercial human models has revealed that angle-based accuracy metrics are insufficient for a variety of reasons. Most tellingly, it is possible to create a wide range of different motions that have similar accuracy on joint-anglebased metrics, but which would result in a large range of ergonomic assessment outcomes for a particular task. The objective of this paper is to delineate the basis for a much broader examination and prioritization of the important aspects of human motions, and hence of human motion simulation for ergonomics.
Some of the observations in this paper are likely to be common knowledge among professional animators, but the current motion-simulation capabilities of the widely used digital human models suggest that the criteria for posture and motion prediction advanced in this paper are not widely understood. Researchers in the computer animation community have examined realism both subjectively and objectively (e.g., Ren et al. 2005). However, the importance of movement fidelity and the relative priority of various aspects of movement for ergonomics analysis has not been described.

\section{METHODS}

The observations in this paper arose from experience in the Human Motion Simulation Laboratory at the University of Michigan, including the development of a number of motion simulation algorithms (e.g., Zhang et al. 1998; Faraway 2004; Park et al. 2004). Studies in the laboratory have included the recording and analysis of tens of thousands of task-oriented human motions, particular seated and standing reaches and object transfers that are similar to industrial tasks often analyzed using human figure models. In a typical study in the laboratory, the motions of ten or more individuals with widely varying body dimensions perform object transfer or reaching tasks while monitored using active and passive motion-capture hardware. The data are analyzed qualitatively by playing the motions back ergonomics software, such as Jack, and in human animation software, such as Poser. Quantitative analyses have been performed at a variety of levels, from kinematics (e.g., Faraway, 2004) to biomechanics (e.g., Dickerson et al. 2001).

One conclusion from this research is that a detailed qualitative examination of real and simulated motions can provide valuable insight to guide the development of simulation algorithms. Even naive viewers can readily detect some types of kinematic inaccuracy in motion simulation. Widespread experience with digital character animation in entertainment, particularly in movies and video games, have increased viewers expectations for realism in simulation. Investigation of these perceptible flaws in motion simulation 
indicates that these visually detected error states often have quantitative consequences for ergonomic analysis. Even when a particular type of inaccuracy does not affect the outcome of ergonomic analyses, the credibility of the analysis is compromised if the simulated motion is perceived as unrealistic.

The application of motion simulation to ergonomic analysis also necessitates greater attention to certain degrees of freedom than others. The purpose of most industrial ergonomics activity is to assess the risk of musculoskeletal disorders, which focuses attention on the most commonly injured body regions, in particular the lower back and shoulder. Consequently, from the perspective of industrial ergonomics, the importance of various aspects of human motion can be prioritized according to their effects on the calculated loading in these body regions.

\section{RESULTS}

\section{Critical Features that are Visually Identifiable}

Critical features are defined as quantifiable aspects of movement simulation that are important either for visual realism or for accurate ergonomic assessments. Most of the critical visual features are important for ergonomics, although not necessarily for every task assessment.

Head/Neck Coordination. People can visually identify an unrealistic head/neck posture, even when such postures differ only slightly, in a quantitative sense, from realistic postures. The appropriate relationship between the head and neck is dependent on how the head and neck linkage is defined relative to the external geometry. An algorithm that assumes a particular neutral-posture relationship between the head and neck will produce unrealistic-looking postures and motions if the neutral posture does not match the neutral posture of the figure to which it is mapped. Hence, prediction algorithms must be flexible enough to simulate motions with respect to a neutral posture rather than globally. Similarly, because the torso posture of the figure can be expected to vary widely during simulations, models of head/neck posture must be defined with respect to the thorax, rather than globally, while taking into account the orientation of the thorax with respect to gravity and the effects of gaze target location.

Environment Contact. "Foot skate" is is a general problem in movement simulation, particular simulations based on motion-capture data (Kovar et al. 2002). When a figure's foot, or indeed any of the part of the body, is in contact with a fixed object (like the floor), the contacting part of the body must remain stationary. For the feet, this means absolutely no movement with respect to the floor while the feet are planted. A hand resting on a table must similarly remain motionless, even while the adjacent limbs are moving. This requirement is difficult to meet unless the model is developed with this constraint in mind. The issue poses a particular problem for joint-angle-based simulation methods, because the endeffector constraint (whether at the foot, hand, or some other part of the body) must be toggled on and off when the constraint becomes active or inactive.
Balance Behavior. People are visually sensitive to out-ofbalance standing postures. Specifically, people seem to understand intuitively that the center of the massive torso must be approximately over the base of support provided by the feet. When hand loads are involved, the visual appearance can be misleading (is the box held in the hands empty or full of heavy parts?), but it is clear that maintaining balance is necessary to meet both visual and biomechanical requirements. When a static posture is out of balance, a movement spanning that posture must include body-segment motions appropriate balance-maintenance. The simplest example is a step in the direction toward which the body is falling. Balance maintenance and step initiation have been studied extensively (e.g., Holbein and Chaffin 1997; Thelen et al. 1997) and rudimentary balance maintenance has been incorporated in human models (Badler et al. 1993), but the findings have not yet been incorporated into a commercial digital human model in a manner that provides visually realistic kinematics for balance-maintenance stepping. A related limitation is that commercial human models are not currently capable of predicting foot placements relative to hand task constraints in a realistic manner.

Critical Feature: Range of Motion Limits. A movement that exceeds normal ranges of motion is visually jarring. For example, if the elbow bends backward, or the head rotates beyond about 90 degrees, the motion is immediately identified as unrealistic. Many movement models explicitly respect joint range-of-motion (ROM) limits, but accomplishing this in a general algorithm that can be applied to many figure models is difficult. Each DHM software package (Jack, Safework, Ramsis, etc.) has different default ranges of motion, and the definitions of joint ROM are inherently tied to the joint-angle definitions each model uses. Interestingly, the intuitive perception of realistic joint motion is apparently keyed not to the average joint ROM typically implemented in DHM, but rather to extreme values, beyond which a posture is seen as unrealistic. This creates a situation in which the default figures in DHM packages typically cannot achieve the range of postures that most individuals can. The problem is particularly acute at the shoulder, where the variation in range of motion in the population is particularly large (Webb Associates 1978).

Interjoint Coordination. Most movement simulations for ergonomic analysis are produced by posture interpolation. Several "keyframe" postures are defined, and the transition between the postures is accomplished by joint-angle interpolation. Quaternion interpolation is available in some software, which reduces some of the anomalies produced by Euler-angle interpolation. Joint angle timing profiles can be applied to produce smooth accelerations and decelerations. The results, while smooth, often do not look realistic, particularly for the extremities, which is where the most flexible joints are located.

A simulated motion in which all or many of the joints in the body start and stop their motions at the same time is often described pejoratively as robotic. An analysis of task-oriented human motions shows many phases of motion, the timing of each of which is critical to achieving a realistic motion. For example, a seated reach to a target might include a phase of 
rapid head and eye motion to acquire the target, a preparatory hand motion while the target is acquired, a relatively slow torso motion to position the upper extremity, a ballistic handmotion phase, and an apparently closed-loop tuning phase as the hand nears the target. While most viewers of a motion could not articulate this sequence, the absence in a simulation of the proper sequencing and overlap of these phases results in an overtly unrealistic motion.

Frequency Content. One near-universal characteristic of simulated motion is an unnatural smoothness. Even motions produced by replaying motion-capture data on an avatar often look unrealistic due to filtering applied to remove noise in the data-capture equipment. In kinematic simulations, the functions used to generate joint-angle trajectories often do not produce sufficient acceleration for movements that a person would make rapidly. The problem is most acute when a person's body comes into contact with a fixed object, such as a foot striking the floor. Foot strike produces relatively high accelerations that are not well simulated in most kinematic models. One solution is to use dynamics models (see Pandy, 2001, for a review) but the complexity, lack of generality, and computational cost of these models have so far limited their application in general-purpose ergonomic tools. Simulations based on kinematics alone can create improved realism by using driving functions that can be tuned to produce the high accelerations that are warranted in particular situations.

\section{Critical Features for Biomechanical Analysis}

Any feature of motion that affects the acceleration of body segments will affect dynamically calculated joint torques. In that respect, all of the visually apparent features discussed above have implications for biomechanical analysis. However, some important features of postures and motion that are not readily apparent visually are nonetheless of critical importance for ergonomic assessments. For example, Chaffin and Erig (1991) showed that an error of 15 degrees in a joint angle can produce as much as a 30 percent error in prediction of population capabilities in high force exertions.

Torso Angle with respect to Vertical. People lift objects with a wide range of torso postures, and biomechanical analyses have shown that torso angle with respect to vertical (gravity) is a major determinant of low-back loading because of the large moment generated by the torso mass. Yet, because people are used to seeing a range of different lifting styles, visual realism for manual materials handling simulations is insufficient to ensure a realistic low-back analysis. Instead, a quantitative metric calculated with respect to gravity, rather than hip angle or other internal measures, is needed to ensure a quantifiable level of accuracy in low-back analysis. The metric should reflect the level of risk imparted to the low back of the simulated person as well as identifying postures that are within the range of those that are likely for a person performing the task.

Arm Extension. When the hands are used to apply force to an object, which is the case in many tasks analyzed with figure models. the moments generated at the shoulder and lower back are often strongly affected by arm extension (how far the hands are from the shoulders). Intuitively, we expect people to bring heavy objects closer to their bodies, to minimize shoulder and low-back moments, but to move lighter objects farther from their bodies when convenient. This aspect of behavior, and the interaction of worker strength and posture, must be incorporated in the simulation if the ergonomic analyses are to be meaningful.

Foot Position. Similarly, the placement of the feet with respect to the hands in standing tasks has a strong influence on both low-back and shoulder moments by determining the range of kinematically feasible postures. In fact, without realistic foot positioning, accurate prediction of torso angle and arm extension may not be possible. Note that foot placement is not well quantified using joint angles, because the same foot placements could be achieved with a wide range of different lower-extremity joint angles.

\section{DISCUSSION}

Every researcher that develops a motion-simulation or posture-prediction algorithm implicitly or explicitly selects a set of critical features. The fitting or validation function that a researcher uses is the quantitative expression of those priorities. This paper argues that metrics calculated from joint angles are not likely to be sufficient to develop good motion simulation algorithms for ergonomic analysis. The identification of two categories of critical motion features also argues for a high-level prioritization of model performance that might involve tradeoffs. For example, one might reasonably compromise on the accuracy of knee-joint angle prediction to achieve good performance in balance maintenance and the prediction of torso angle with respect to gravity. Of course, achieving these high-level goals is likely to be accompanied by acceptable performance on subsidiary degrees of freedom.

The selection of an approach to modeling human motion should be guided by a careful consideration of the uses to which the model will be applied. Because ergonomic analyses rely extensively on calculations of joint moments, accurate predictions of shoulder and low-back moments should be given priority over other factors. Model development will also benefit from the recognition that the human ability to recognize unrealistic motion can be exploited to identify the critical features of good motion simulation.

\section{REFERENCES}

Badler, N.I., Phillips, C.B., and Webber, B.L. (1993). Simulating Humans. Computer Graphics, Animation, and Control. Oxford University Press.

Chaffin, D.B., and Erig, M. (1991). Three-dimensional biomechanical static strength prediction model sensitivity to pos and anthropometric inaccuracies. IIE Transactions, 22(3):215-22

Dickerson, C.R., D.B. Chaffin, K. Kim, B.J. Martin, and N. Womack. (2001). The Effect of Motion Dynamics in Calculation of External Joint Moments During Light Industrial Hand Motions. Proceedings of American Society of Biomechanics, San Diego, CA. 
Faraway J. (2004). Human Animation using Nonparametric Regression Journal of Computational and Graphical Statistics. 13:537-55

Holbein, M.A. and Chaffin, D.B. (1997). Stability Limits in Extreme Postures: Effects of Load Positioning, Foot Placement, and Strength. Human Factors, 39(3):456-468.

Kovar, L., Gleicher, M., and Schreiner, J. (2002). Footskate cleanup for motion capture editing. Proc. ACM SIGGRAPH Symposium on Computer Animation.

Pandy, M.G. (2001). Computer Modeling and Simulation of Human Movement. Annual Review of Biomedical Engineering, Vol. 3: 245-273.

Park, W., Chaffin, D.B., and Martin, B.J., (2004). Toward memory-based human motion simulation: development and validation of a motion modification algorithm. IEEE Transactions on Systems, Man, and Cybernetics, Part A: Systems and Humans, 34 (3), 376-386.

Ren, L, Patrick, A., Efros, A.A., Hodgins, J.K., Rehg, J.M. (2005). A Data-Driven Approach to Quantifying Natural Human Motion, ACM Transactions on Graphics (SIGGRAPH 2005).

Thelen, D.G., Wojcik, L.A., Schultz, A.B., Ashton-Miller, J.A., Alexander, N.B., (1997). Age differences in using a rapid step to regain balance during a forward fall. Journals of Gerontology Series A: Biological Sciences and Medical Sciences, Vol 52, 8-13

Webb Associates (1978). NASA Anthropometric Sourcebook, Vol. 1. NASA Ref. 1024. NASA, Washington, D.C.

Zhang, X., A.D. Kuo, and D.B. Chaffin. Optimization-based differential kinematic modeling exhibits a velocity-control strategy for dynamic posture determination in seated reaching movements, Journal of Biomechanics, 31:1035-1042, 1998. 